\title{
GLAD!
}

Revue sur le langage, le genre, les sexualités

05 | 2018

Raconter les sexualités depuis la marge

\section{Linguistique queer, sexualité et analyse du discours}

Queer Linguistics, Sexuality, and Discourse Analysis

\section{William L. Leap}

Traducteur : Sara Martinez

\section{CpenEdition}

\section{Journals}

Édition électronique

URL : http://journals.openedition.org/glad/1244

ISSN : 2551-0819

Éditeur

Association GSL

Référence électronique

William L. Leap, «Linguistique queer, sexualité et analyse du discours », GLAD! [En ligne], 05 | 2018, mis en ligne le 15 décembre 2018, consulté le 17 décembre 2020. URL : http://journals.openedition.org/ glad/1244

Ce document a été généré automatiquement le 17 décembre 2020.

\section{(c) (i) (9)}

La revue GLAD! est mise à disposition selon les termes de la Licence Creative Commons Attribution Pas d'Utilisation Commerciale - Pas de Modification 4.0 International. 


\section{Linguistique queer, sexualité et analyse du discours}

Queer Linguistics, Sexuality, and Discourse Analysis

William L. Leap

Traduction : Sara Martinez

\section{RÉFÉRENCE}

LEAP, William L.. 2011. « Queer linguistics, sexuality, and discourse analysis » in The Roudledge Handbook of Discourse Analysis, GEE, James Paul \& HANDFORD, Michael (éds). Londres et New-York : Routledge, 558-571.

1 La linguistique queer est un domaine de recherche universitaire relativement récent, mais qui s'est taillé une place de choix dans les débats en cours sur le langage, le genre et la sexualité. Tout comme les recherches linguistiques sur le genre (voir Coates 2011), la linguistique queer refuse d'aborder les pratiques linguistiques à travers le prisme d'une prétendue binarité homme/femme (ou encore à travers un choix réduit d'identités que l'on associe à des pratiques et préférences sexuelles, telles que homo/ hétéro). Au contraire, elle met au jour les présupposés qui mènent les chercheurs à considérer le genre comme un système prédéterminé et figé.

2 Le terme "queer » est un angle d'approche particulièrement adapté à cette démarche. Ici, comme partout dans la théorie queer:

queer ne désigne aucune espèce naturelle et ne se réfère à aucun objet déterminé; il prend son sens dans sa relation d'opposition à la norme. (Halperin 2000 [1995] : 76)

3 La linguistique queer postule que le genre est la référence normative de ce débat, et que les positions d'une société donnée sur "qui sont les hommes, qui sont les femmes » sont des constructions idéologiques; elles constituent une « représentation du rapport imaginaire des individus à leurs conditions réelles d'existence", qui a pour fonction « de constituer des individus concrets en sujets » (Althusser 2011 [1970] : §139, 176). Ces 
relations se présentent aux individus par le biais de pratiques discursives, mais elles le font différemment en fonction de leurs caractéristiques raciales et ethniques, de leur position sociale, de leur âge, de leur (in)validité, ainsi que de leur citoyenneté, de leur nationalité et de leur situation dans les flux migratoires, autant d'éléments qui façonnent les débats actuels sur le genre. Dans ce contexte, s'il est vrai que la force de conviction des constructions idéologiques subsiste, il est peu probable que les conceptions courantes relatives aux hommes et aux femmes soient homogènes; au contraire, elles constituent souvent des motifs de désaccord et de conflit.

4 En étudiant les pratiques discursives par le biais desquelles se déploient ces points de vue, ces désaccords et ces conflits, la linguistique queer confirme les limites du genre en tant que catégorie explicative de l'analyse linguistique et contraint les recherches relatives à «qui sont les hommes, qui sont les femmes » à aller au-delà de ces discours de certitude. À ce stade, le terrain de la sexualité devient un autre espace d'investigation, car les « relations d'opposition» associées aux normes de genre sont souvent situées dans, et médiées par les pratiques, les identités et les désirs sexuels. Quoi qu'il en soit, la linguistique queer ne peut pas être axée uniquement sur la sexualité, pas plus qu'elle ne peut l'être sur le genre. Les pratiques, les identités et les désirs sexuels sont également modulés de diverses façons sur le plan social; dans certains cas, ils se conjuguent dans l'intimité ou le silence plutôt qu'en tant qu'éléments d'un discours public. Consciente que certains messages idéologiques sur «qui sont les hommes, qui sont les femmes » circulent à grande échelle dans la société actuelle, la linguistique queer explore les déclinaisons discursives du sexuel - désirs, pratiques et identités - sous l'angle de leur déploiement dans ces circulations et souvent (comme l'indique Halperin) en opposition à cette circulation.

5 En résumé : si, comme Judith Butler, nous estimons que le genre est « une série d'actes répétés à l'intérieur d'un cadre régulateur des plus rigides, des actes qui se figent avec le temps de telle sorte qu'ils finissent par produire l'apparence de la substance » (2006 [1990] : 110), alors « quel genre de répétition subversive pourrait remettre en question la pratique régulatrice de l'identité »? (op. cit. : 108). La linguistique queer s'intéresse aux pratiques discursives dans le but de répondre à la question de Butler.

\section{Lire de manière queer les discours sur la sexualité : un exemple}

6 J'aborde à présent un exemple montrant ce que pourrait être une analyse linguistique queer des pratiques discursives - sexualité, genre, identité et sujets connexes. La situation présentée dans cet exemple est extraite d'un récit de vie que j'ai collecté dans le cadre d'un travail sur le terrain (1995-1999) dans la région métropolitaine du Cap, en Afrique du Sud. Les changements en matière de géographie sexuelle urbaine qui avaient lieu au moment de l'abandon des politiques «d'apartheid strict » et du passage à des processus démocratiques étaient particulièrement intéressants. Pendant la période de recherche, j'ai mené des entretiens avec des hommes homosexuels blancs vivant en centre-ville et en banlieue ainsi qu'avec des hommes homosexuels coloured ${ }^{1}$ et noirs vivant dans les townships des Cape Flats ${ }^{2}$. Ces récits m'ont appris que la vie homosexuelle avait été extrêmement touchée par la ségrégation au Cap sous l'apartheid. Mais j'ai également découvert que dès 1990, les lois de l'apartheid avaient commencé à s'assouplir dans certains quartiers commerçants du centre-ville, pour 
répondre aux besoins des voyageurs internationaux. La loi autorisait que dans la zone du Cap, les personnes non blanches puissent désormais accéder à ces lieux, et certaines d'entre elles essayèrent de profiter de ces occasions. Néanmoins, c'était un strict apartheid qui régnait dans les quartiers d'affaires du centre-ville, et certains propriétaires de clubs firent en sorte de perpétuer l'exclusion raciale en dépit des changements législatifs.

7 Les commentaires dans ces récits de vie, tels que l'exemple 1 ci-dessous, indiquent que certains homosexuels coloured et noirs étaient conscients des situations paradoxales survenant dans ce contexte. Ils comprenaient bien que les pratiques d'exclusion faisaient d'eux des sujets rejetés, et que dans le même temps, ces premiers changements dans la politique d'apartheid rendaient enfin envisageable la fin des discriminations. Jameson, le narrateur de l'exemple 1, était âgé de 35 ans au moment de notre entretien (1996) et avait remarqué pour la première fois ces contradictions alors qu'il approchait la trentaine. Il a grandi dans l'un des townships coloured du Cap, et vivait encore chez ses parents. Lorsqu'il m'a raconté son histoire, il travaillait pour un service social du township. C'est un ami commun qui nous a présentés ; il lui avait parlé de mon intérêt envers la géographie sexuelle du Cap. Jameson m'a parlé très volontiers, car il avait beaucoup d'histoires à raconter sur l'homosexualité et les pratiques topographiques liées aux townships et au centre-ville. L'exemple 1 est l'une de ces histoires.

\section{Exemple 1}

$001 \mathrm{~J}$ : ... j'ai fini par aller [à la gare de Rondebosch] régulièrement et ensuite 002 j'ai rencontré quelqu'un là-bas. À la gare. Je le connaissais de la fac et heu on

003 a commencé à discuter, et tous les vendredis on se voyait là. Je lui ai donné mon numéro de téléphone

004 et il m'a donné le sien, je lui ai dit on se retrouve à Rondebosch à telle heure. On sortait draguer

005 dans le quartier, certains soirs on avait de la chance, d'autres moins.

006 Un jour il a parlé de ce bar gay du Cap. Il a dit allons-y, j'ai

007 dit pas question que j'aille dans un bar gay. J'avais la trouille, j'étais très 008 fatigué, j'étais sorti à onze heures pour aller à la gare.

009 Puis un soir il a dit allons-y, on verra ce que ça donne. On y est allés, c'était

010 rempli [dit dans un murmure excité] d'hommes! Les gens vous regardaient

011 et se frottaient à vous en passant, et j'ai pensé, c'est mon truc. Et puis on est partis. Et la

012 semaine d'après, on a dit, ok, maintenant on connaît l'endroit, on $\mathrm{y}$ retourne ou quoi ? 013 On y est retournés et c'était fermé.

(WL : C'était où, exactement ?)

$014 \mathrm{~J}$ : C'était le [endroit 1]. C'était fermé. J'ai pensé oh non, où est passé le bar?

015 Mon ami, bien sûr, s'est renseigné auprès d'autres personnes, ils nous ont dit qu'un nouvel endroit avait

016 ouvert, le [endroit 2]. On a fini par trouver [endroit 2] et on

017 y est allés. Le premier soir, on est entrés et on a pris un verre, on est restés environ

018 une heure et puis on est partis.

019 La semaine d'après on y est retournés, et ils nous ont dit, vous ne pouvez pas entrer, c'est réservé

020 aux membres. On a demandé, comment ça, les membres ? On est venus la semaine dernière. Non, c'est juste pour 
021 les membres. Le comptoir est ouvert au public, et l'arrière-salle aussi. Là, on a

022 compris que c'était une question raciale et heu, on s'est assis au comptoir

023 et on s'est sentis rejetés parce qu'on s'était bien amusés la semaine précédente. Et on a demandé au barman

024 pourquoi on ne peut pas entrer, et il nous a demandé : "Vous êtes homos ? " À ce moment-là, aucun de nous deux

025 ne voulait admettre ça, alors on a répondu « quelle importance ? » Il a

dit, c'est

026 un bar gay, donc c'est très important.

027 On est partis, et on est revenus la semaine d'après ; on a décidé d'aller

dans la partie du bar

028 réservée aux membres, on est entrés, et heu personne ne nous a arrêtés.

029 Et le barman a dit que [endroit 3], c'était le bar où on devrait aller. Et

donc

030 c'est comme ça que j'ai découvert la scène des bars.

(Notes de terrain de W. Leap)

$8 \mathrm{Si}$ on le prend au pied de la lettre, l'exemple 1 correspond à un récit homosexuel classique : c'est une histoire de coming out, imbriquée dans une histoire de découverte sexuelle/topographique. Au départ, d'après ce récit, Jameson ne connaissait pas les établissements homosexuels de la ville, et se voit contraint de rencontrer des hommes en draguant dans les cottages (toilettes publiques) et le parking de la gare ferroviaire de Rondebosch ${ }^{3}$. Ce processus lui permet de confirmer l'identité homosexuelle d'un homme qu'il a rencontré à l'université, devenu son partenaire dans cette exploration urbaine. L'ami a suggéré la première incursion en centre-ville, et en tâtonnant, ils ont fini par trouver plusieurs clubs, négocié leur entrée, et ainsi « découvert la scène des bars » $(1: 030)$. Le processus de découverte a comporté un moment difficile. Jameson et son ami ont été exclus d'un club en raison de ce qu'ils ont compris être « une question de race » (1:021-022). Mais le barman voyait les choses différemment. Il a demandé à Jameson : «Vous êtes homos? » et lui a demandé de faire une déclaration de coming out, que celui-ci n'était pas prêt à faire.

9 Jameson a donc remanié la paire adjacente anticipée - «Quelle importance ?» - et a brusquement quitté les lieux ; il y est retourné plus tard et a cherché à y entrer dans un contexte différent, avec succès cette fois-ci.

10 Considérer l'exemple 1 comme un récit de coming out met l'accent sur le fait que les événements présentés ont précipité la formation de l'identité sexuelle du sujet. Cette interprétation ne reconnaît pas entièrement la signification de la question raciale, ou la mesure dans laquelle l'apartheid, même au début de la transition, pourrait avoir modelé la construction sexuelle en termes raciaux. Le fait que Jameson et son ami se voient refuser l'entrée du club privé pour une «question raciale» (1: 021-022) constitue bien sûr un élément important à cet égard. Mais il faut garder en tête que Jameson et son ami avaient déjà accédé au club privé et y ont été admis lors de leur visite suivante. Visiblement, à ce stade de l'histoire du Cap, la pratique d'exclusion raciale ne consistait plus à refuser catégoriquement les non-blancs: il s'agissait désormais de les exclure de manière arbitraire et imprévisible. Dans ce contexte, le récit de coming out et de découverte particulière/sexuelle de l'exemple 1 devient une histoire de formation du sujet rejeté, ainsi qu'une histoire illustrant la façon dont deux 
sujets ont remis en cause les conditions contradictoires de refus une fois que la formation du sujet en ces termes a commencé de se déployer.

11 Selon cette interprétation, la question du barman : «Vous êtes homos? » $(1: 204)$ revêt une signification particulière. Comme Jameson l'a expliqué :

... [on s'est] assis au comptoir, on s'est sentis vraiment rejetés parce qu'on s'était bien amusés la semaine d'avant. Et on a demandé au barman pourquoi on ne pouvait pas entrer. $(1: 022-024)$

12 La réponse de Jameson à la question du barman montrait une réticence à faire une déclaration publique quant à leur sexualité, comme il l'a expliqué :

À ce moment-là, aucun de nous deux ne voulait admettre qu'on était homos, alors on a répondu «Quelle importance? » Il a dit, c'est un bar gay, donc c'est très important. $(1: 024-026)$

13 Mais la réponse de Jameson va au-delà de ça : la question du barman sous-entendait que Jameson et son ami pouvaient accéder au club privé s'ils admettaient qu'ils étaient homosexuels. Répondre «oui » à sa question n'aurait pas changé le fait qu'ils avaient été préalablement refoulés pour une question raciale, mais cela leur aurait permis d'accéder au club privé et aurait aboli leur statut de sujets rejetés. Dans le même temps, répondre "oui » serait également revenu à admettre que l'homosexualité efface les différences raciales dans le milieu gay de la ville, que les homosexuels de couleur avaient désormais le droit de " passer pour des blancs » dans le contexte d'un apartheid finissant. En répondant "non" à la question du barman, Jameson et son ami ont préservé l'intégrité de leur statut racial même si, ce faisant, ils ont conservé le statut de sujets rejetés, maintenus à distance des lieux de rencontres sexuelles qui les intéressaient grandement dans un premier temps. En assumant ce statut, Jameson et son ami ont également mis en évidence la position idéologique exprimée dans l'acte de refus du portier: "gay", c'est implicitement un statut de blanc, que les personnes racisées acquièrent uniquement dans un contexte spécifique ou en tout cas des circonstances exceptionnelles.

14 Il n'est donc pas malvenu de considérer l'exemple 1 comme un récit de coming out, mais le réduire à ce genre oriente les éléments pertinents de l'histoire racontée. En outre, comme le révèle une lecture du texte plus attentive, la portée de ces éléments pertinents s'avère bien plus complexe qu'une trajectoire de découverte de soi. De même, l'exemple 1 pourrait être qualifié de récit « gay » dans le sens où le narrateur est homosexuel, se qualifie d'homme gay et raconte des expériences (coming out, découverte) dont les homosexuels de maints endroits du monde sont familiers. Mais encore une fois, le terme "gay » ne peut avoir de sens prédéterminé dans le contexte de ce récit. En réalité, l'un des éléments de la «scène des bars» que Jameson a "découverte» $(1: 030)$ en s'aventurant dans les établissements commerciaux du centre-ville a été ce que la catégorie "gay » pouvait signifier pour lui à cette époque précise de l'histoire sociale et politique du Cap. 


\section{La linguistique queer en tant qu'élément d'un « projet queer " plus vaste}

Enfin, le tissage continu entre sexualité, race et réponses au système d'apartheid que décrit l'exemple 1 peut également être compris comme un cas de "répétition subversive » dont les effets « pourrai[en]t remettre en question la pratique régulatrice de l'identité » (Butler 2006 [1990] : 108). Il est important de dire que la répétition et la subversion ne sont pas des propriétés « $d u$ » texte, mais reflètent plutôt les engagements des sujets parlants et écoutants dans leur activité de production et de réception des discours: Jameson et son ami, le portier, le barman, mais aussi le chercheur - et à présent le lecteur de cet article - se voient profondément impliqués dans le processus de formation du sujet rejeté tel que décrit dans le texte. Cela constitue une raison supplémentaire de considérer que l'exemple 1 n'est pas simplement un récit de coming out et de découverte sexuelle/topographique ni un autre type d'histoire gay. Ce qui est en jeu ici, ce sont les modulations irrégulières entre la pratique discursive et les structures de pouvoir, la sexualité étant si souvent utilisée comme signal des points critiques d'intersection: "On a compris que c'était une question de race... [mais] le barman a demandé : "Vous êtes homos?” (1: 021-022, 024).

16 La théorie queer, dans son ensemble, s'intéresse également aux modulations irrégulières entre la sexualité, les pratiques discursives et les structures de pouvoir. De même que la linguistique queer, la théorie queer se penche «non seulement sur ce qui est dit, mais aussi sur le contexte dans lequel les récits se déploient» (Giffney 2009 : 7). En fait, comme Giffney l'explique, la théorie queer elle-même peut être qualifiée d'« exercice d'analyse du discours" dans le sens où «[elle] prend très au sérieux la signification des mots et le pouvoir du langage » $(2009: 7)$.

$17 \mathrm{Au}$ début d'Épistémologie du placard, l'un des textes fondateurs du corpus queer, Eve Sedgwick écrit :

[...] la culture occidentale moderne [a] placé ce qu'elle appelle «sexualité » dans une relation de plus en plus étroite avec nos principales conceptions de l'identité individuelle, de la vérité et du savoir... [et] le langage de la sexualité non seulement croise mais transforme les autres langages et relations par lesquelles nous produisons de la connaissance ». (Sedgwick 2008 [1990] : 25)

18 La théorie queer ne s'est pas contentée de débattre de la question « du sexuel » : elle est née en observant les façons dont la « sexualité, en tant que catégorie nommée, impose de la signification à - et donc contribue à réguler - d'autres dimensions de l'expérience humaine. Ainsi, la recherche queer cherche depuis longtemps à proposer :

une compréhension plus profonde des structures discursives et des systèmes de représentation qui déterminent la production des significations sexuelles et qui organisent les perceptions individuelles de telle sorte qu'elles perpétuent et reproduisent les fondements du privilège hétérosexuel. (Halperin 1995 [2000] : 47)

19 Il faut reconnaitre - et la discussion sur l'exemple 1 l'a confirmé - qu'il existe des formes de pratiques discursives non langagières, qui contribuent à «déterminer la production des significations sexuelles et [...] microgèrent les perceptions individuelles » en matière de sexualité et de privilège hétérosexiste. Pour cette raison, et : 
étant donnée sa volonté d'interroger les processus sociaux qui ont non seulement produit et reconnu mais également normalisé et entretenu l'identité, la promesse politique du [queer] a spécifiquement résidé dans sa lecture élargie de multiples antagonismes sociaux, tels que la race, le genre, la classe, la nationalité et la religion, outre la sexualité. (Eng et al. $2005: 1$ )

Au fil du temps, la « critique globale » de la " production des significations sexuelles » et des "multiples antagonismes sociaux » y attenant a revêtu une grande diversité de formes : théorie critique de la race et queer critique of coulour, crip theory ${ }^{4}$, recherches sur la transidentité, nouvelles propositions de la théorie post-coloniale, recherches sur les migrations/la diaspora, recherches sur l'(homo)phobie/les discours de haine. La linguistique queer apporte ses idées à cette "lecture élargie ", et dans les parties suivantes, j'étudie certains de ces points de vue et les projets sur lesquels ils sont basés.

\section{La linguistique queer sur sa propre voie : de l'identité à la performativité et au désir}

21 La linguistique queer est née à la fin des années 1990 en tant que critique des recherches " assimilant ce que certains hommes homosexuels (là encore, généralement blancs, de la classe moyenne et éduqués) disent dans certains contextes avec un "anglais gay" général» (Kulick 1999: 616) et d'autres travaux postulant que les pratiques linguistiques indexaient l'identitié genrée et/ou le corps sexué. Plutôt que d'affirmer que le parler gay présentait certaines propriétés indexicales dans ces situations-là, la linguistique queer a préféré problématiser ces propriétés.

22 Comme Livia et Hall l'ont expliqué, il n'était pas suffisant d'avancer que certaines formes d'énonciation décrivent non seulement le monde mais agissent sur celui-ci une manière de "faire des choses avec des mots» (1997: 11). Ce qui rend l'effet performatif possible dans chaque situation ne peut pas toujours être nommé, et les tentatives de mettre le doigt sur des particularités linguistiques faisant de certains textes ou de certains actes des textes et des actes purement queer ont généralement échoué.

23 La «dimension queer» de pratiques linguistiques vient autant de la réponse d'un interlocuteur à une pratique linguistique que de toute représentation formelle que les énonciateurs donnent au message ou à la signification souhaités.

L'exemple 2, issu du monologue oral d'une drag queen afro-américaine pendant son spectacle dans un cabaret d'Austin (Texas), montre la façon dont la construction d'un message sexuel dépend d'une interaction subtile entre l'intention du locuteur et la reconnaissance de l'interlocuteur.

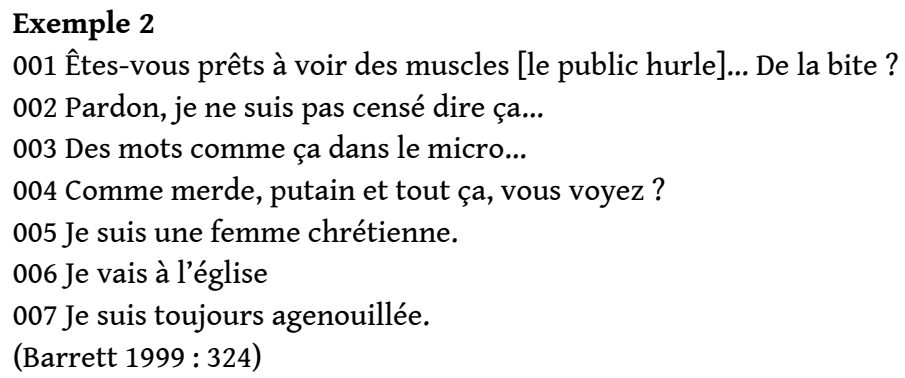


Comme le fait remarquer Barrett, le performeur a adopté une «façon de parler de femme blanche » (FB, ci-après), calqué sur le comportement des femmes blanches et les pratiques discursives correspondantes décrites par Lakoff (1975 : 56-56). L'utilisation du discours FB par sa persona de drag queen encode l'intention du performeur de se présenter sur scène en femme distinguée de la classe moyenne (Barrett, 1999: 321). Mais le drag consiste notamment à être plus vrai que nature, à surpasser et à critiquer plutôt qu'à imiter l'objet de la performance. La contradiction entre l'incarnation afroaméricaine du performeur et sa pratique verbale de «femme blanche » illustre la chose suivante: si un homme noir peut "être " une femme blanche sur la scène du cabaret drag, alors quelqu'un d'autre peut " passer pour blanc ", et par conséquent la catégorie «femme blanche » commence à perdre son apparence d'autorité. L'introduction par le performeur d'une posture discursive d'opposition vient également souligner ce phénomène. À la ligne $2: 001$, le performeur emploie un terme vulgaire pour parler des organes génitaux masculins qu'une femme distinguée de la classe moyenne serait peu susceptible d'utiliser en public. À la ligne $2: 004$, il ajoute des jurons supplémentaires, tout aussi incongrus. Le discours FB revient à $2: 005-006$, avec une allusion qui situe le raffinement $\mathrm{FB}$ dans un christianisme performatif, tandis que la ligne $2: 007$ prolonge ou subvertit ces allusions, selon la réaction du public qui interprète la remarque dans un sens pieux ou érotique.

26 Le fait est que le performeur ne dit à aucun moment de manière textuellement explicite "Je suis une drag queen». Ce message est encodé dans l'accumulation de matériaux discursifs du passage rapporté ; mais cette accumulation doit être examinée en relation avec l'incarnation du performeur dans le contexte de la performance et en opposition avec les déclarations contradictoires que déploient les matériaux discursifs eux-mêmes. Comme dans l'exemple 1, la sexualité est un élément par le biais duquel la mise au travail de la dimension queer s'exprime et, comme dans l'exemple 1 également, le texte doit être interprété dans un sens large et non pas de manière fermée.

27 Dans ces conditions, les débats portant sur les pratiques linguistiques étroitement liées à l'identité sexuelle - comme la discussion sur «l'anglais gay » chez Leap (1996) et d'autres écrits connexes - ont dû être rethéorisés, si ce n'est entièrement rejetés. Cameron et Kulick (2003b: 75-105, voir également Kulick 2000) ont formulé des propositions particulièrement solides en ce sens. Ils ont critiqué les recherches sur le parler gay et lesbien et tout projet qui « resterait dans l'idée que le " parler queer » est d'une façon ou d'une autre lié aux identités queer (c'est-à-dire non hétérosexuelles) » (2003 : 102) : la focalisation sur l'identité sexuelle, quelle que soit son orientation, «n'étudie en rien tout ce qui constitue probablement la sexualité, à savoir les fantasmes, la répression, le plaisir, la peur et l'inconscient " (2003: 105). Cameron et Kulick ont plutôt proposé que "le language et le désir » deviennent le point d'entrée des recherches sur l'articulation entre language et sexualité.

Ce changement de perspective "reconnaît que la sexualité est centrée sur l'érotique » et indique "dans quelle mesure nos vies érotiques sont façonnées par des forces qui ne sont pas totalement rationnelles et dont nous ne sommes pas pleinement conscients $(2003: 106,107)$.

28 L'assimilation de la sexualité au " désir ", « désir » étant un terme parapluie désignant " les fantasmes, la répression, le plaisir, la peur et l'inconscient », a élargi les horizons des recherches sur la queerness comme un « devenir » libéré de toute notion d'identité. Mais il restait à savoir si la linguistique queer pouvait fonctionner dans ce cadre. 
Certains chercheurs ont avancé que consacrer entièrement l'analyse linguistique à ce type d'enquête décontextualisée sépare artificiellement la sexualité de l'économie et de l'histoire politiques, ce qui débouche sur ce que Penelope Eckert a appelé «la mystification » de l'étude de la sexualité (2002: 100) :

Le défi consiste désormais à adopter une approche axée sur la médiation sociale du désir : construire une conception du désir qui soit à la fois interne et individuelle et externe et partagée. (Eckert $2002: 100$ )

La discussion de Cameron et Kulick sur le langage et le désir répondait directement au « défi » d'Eckert ${ }^{5}$.

Bien qu'il soit possible d'expérimenter le désir sexuel en tant que phénomène purement personnel et intensément privé, sa forme est façonnée par les interactions sociales et verbales. (2003b:131)

Reconnaître que le désir est une construction "socialement médiée " a révélé deux choses quant à la relation entre le langage et le désir (ainsi que sur le langage et la sexualité, plus largement). En premier lieu, le désir est spécifiquement :

rendu intelligible [dans le langage] parce qu'il fait appel à des codes de signification qui circulent dans la société au sens large - pour reprendre les termes d'Eckert, il est «extérieur et partagé » [et, deuxièmement] «les individus ne peuvent pas refuser que leurs désirs soient compris en termes de normes sociales dominantes ». (Cameron and Kulick 2003b : 132)

Bien qu'elle n'y fasse pas directement référence, cette déclaration reconnaît l'intérêt pour le langage et l'idéologie qui a été au cœur de l'analyse du discours pendant quelques années (voir Fairclough 2011 et Van Dijk 2011), intérêt qui est devenu partie intégrante des recherches sur le langage et la sexualité dans d'autres pans de la théorie queer. Cela dit, les processus idéologiques qui intéressaient alors les chercheurs n'ont pas pu être décrits simplement comme interpellant ou « hélant » la forme du sujet. Les idéologies sexuelles promeuvent dans certains cas l'obéissance des sujets mais elles peuvent également favoriser les conditions de «désidentification» (Muñoz 2000: 11-34) dans lesquelles les locuteurs s'écartent du terrain normatif et «travaillent » la forme du sujet qui leur a été imposée par l'idéologie. Ce faisant, ils forcent l'idéologie à " fonctionne[r] en quelque sorte à l'envers, c'est-à-dire sur et contre elle-même, à travers le "renversement-remaniement" du complexe des formations idéologiques (et des formations discursives qui s'y trouvent intriquées) » (Pêcheux $1975: 201$ ).

32 Parce que les sujets transgenres sont si fréquemment pris dans un travail de désidentification, les relations entre le transgenre et le langage sont devenues un domaine de recherche particulièrement fructueux de la linguistique queer. À leurs débuts, les recherches sur le " parler transgenre » cherchaient à évaluer la façon dont des personnes transgenres se conformaient aux normes linguistiques de leur genre choisi. La recherche en linguistique queer a immédiatement remis en question ce type d'études qui s'appuient sur une conception limitée des pratiques langagières, et a développé une forte critique de l'obligation de conformité que ces études présupposaient.

33 Comme Valentine (2007) l'a montré, une partie du problème réside dans la popularité croissante du terme «transgenre » lui-même. Si ce terme prétend désigner des sujets conformément à leur " genre d'identification et non au genre qui leur a été assigné à la naissance", il est aussi devenu "une abréviation utile désignant des genres non normés de manière générale », et donc " une façon de décrire un groupe de personnes diverses à la fois aux États-Unis et au-delà » $(2007: 23,19)$. Dans ce sens, «transgenre » 
est une formation artificielle, qui décrit par un seul terme toute une gamme d'expériences dont les détails se voient désormais brouillés par les effets unifiants de la catégorie. De même, alors qu'un éventail de pratiques linguistiques peut être associé à l'expérience transgenre, il est tout aussi artificiel de penser que ces pratiques constituent un "parler transgenre » unique, puisque les sujets transgenres mobilisent une série de pratiques linguistiques ainsi que d'autres types d'encodages discursifs pour signifier leurs droits à un genre ou à une identité sexuelle différents de ceux qui leur ont été assignés à la naissance.

34 Certaines pratiques linguistiques, par exemple, permettent aux locuteurs de faire état d'un statut transgenre dans le cadre d'un discours oral; ces derniers peuvent "détourner les règles" qui gouvernent généralement les fonctions de marquage du genre des adjectifs, des pronoms, des terminaisons de verbe, jouer avec des références métaphoriques ou encore manipuler d'autres éléments de la structure linguistique de surface (Kulick 1998 : 206-211; Morial 1998; Hall 2002). Mais il existe également le " stealth ${ }^{6}$ ", une synthèse créative de choix de mots et d'intonations, de postures et de gestes, de styles vestimentaires et d'autres marqueurs permettant aux sujets trans opérés d'encourager une lecture normative plutôt que transgenrée de leur incarnation, ce qui leur évite ainsi de se voir interroger sur leur genre de naissance (« Il l'est ou il ne l'est pas? ») avant que ces questions potentiellement embarrassantes ne soient posées en public (Edelman 20097). La linguistique queer explore la façon dont les locuteurs ont recours à ces pratiques, à quel moment ils le font, et qui le fait. Elle étudie également les formes prises par la réception par les destinataires du message que ces pratiques entraînent, ainsi que les messages sur le genre (local et idéologique) qui se voient validés et/ou contestés au cours de ces processus.

\section{Intentionnalité, (inter)subjectivité, appartenance et citoyenneté}

Un certain degré d'intentionnalité du locuteur était impliqué dans ces discussions sur la transidentité et le langage. Cela a constitué un thème fondamental des premiers débats sur la langue et l'identité gay (Leap 1996: 24-73) ainsi que de la première critique queer de la performativité (Livia et Hall 1997 : 11). Mais l'intentionnalité était inenvisageable dans le cadre des recherches sur le langage et le désir telles que Cameron et Kulick les menaient à l'origine, car les décisions conscientes qu'un locuteur donné pourrait avoir pendant une pratique textuelle pourraient toujours être éclipsées par l'échec, la contrefaçon, le détournement ou d'autres caractéristiques de l'itération (Cameron et Kulick 2003a; Kulick 2003 : 112-122).

36 Pour résoudre ce problème, Bucholtz et Hall (2004) sont allés au-delà du travail du locuteur et ont considéré "l'identité, sexuelle et autre» comme "le résultat de pratiques et d'idéologies négociées de manière intersubjective»(2004:4698). Les pratiques linguistiques sont profondément intégrées à chaque niveau de ces négociations intersubjectives. Mais plutôt que d'être issues d'un répertoire prédéterminé, la forme que revêtent ces pratiques et les fonctions qu'elles remplissent sont façonnées par l'engagement dynamique des locuteurs et des interlocuteurs.

La discussion de Barrett de la performance de la drag queen afro-américaine (exemple 2) illustre parfaitement la façon dont «les tactiques d'intersubjectivité » se 
déploient, particulièrement la manière dont elles acquièrent une représentation dans la pratique linguistique via des connexions polyphoniques entre des formes spécifiques de genre linguistique, dont l'afro-américain vernaculaire et le "parler de femme blanche». Brian King (2008) abonde dans ce sens lorsqu'il montre que le désir homosexuel a encouragé l'apprentissage de l'anglais chez des hommes coréens vivant en Nouvelle-Zélande. Les hommes interviewés par King ont fait état de fréquents sentiments de malaise lorsqu'ils parlaient anglais avec des personnes blanches, mais ont également remarqué que cet embarras disparaissait lorsqu'ils parlaient cette langue avec des hommes blancs homosexuels. King cite par exemple le cas de Hyoung, un homme de 35 ans, issu de la classe moyenne et se déclarant homosexuel, qui a indiqué faire tout son possible pour se tenir à distance de la communauté coréenne locale, car "comme je suis GAY, ils voulaient savoir où j'étais et tous les détails " (2008: 241). Il préférait donc fréquenter le réseau social de son petit ami (non coréen). King a demandé à Hyoung s'il était "à l'aise avec tous ces gens? pour parler// anglais? ». L'exemple 3 montre les réponses de Hyoung.

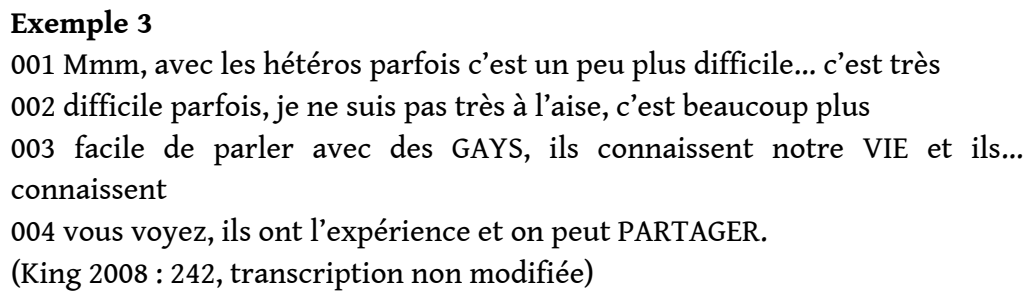

Hyoung renvoie ici à une sexualité discursive modelée en réponse aux tensions entre le même et le différent, que Bucholtz et Hall (2004: 494) appellent «tactiques d'adéquation et de distinction". Ici, néanmoins, la mêmeté et la différence ne coïncident pas avec les contrastes linguistiques au sens large entre l'anglais et le coréen, mais indiquent une lutte plus nuancée pour établir leur place sur chaque terrain linguistique et social. Autrement dit, selon son propre compte rendu, Hyoung lutte avec d'autres personnes de la communauté coréenne locale pour démontrer sa conformité aux attentes de l'idéologie hétéronormative. Son sentiment de différence est renforcé lorsqu'il se trouve dans ces situations, même si les pratiques discursives (coréennes) lui sont par ailleurs familières. Lorsqu'il est en compagnie d'« homosexuels", toute incertitude que lui et d'autres pourraient avoir quant à son statut sexuel se voit balayée par les attentes de l'expérience gay commune et par la familiarité supposée avec des pratiques linguistiques par le biais desquelles ces attentes sont encodées. Cependant, il s'agit là de compétences linguistiques en anglais, une langue que Hyoung commence tout juste à maîtriser. Il est donc confronté à un autre type de difficulté linguistique, mais cet obstacle est amoindri par la cordialité du contexte social.

39 L'anglais devient une sorte de "langue gay » dans l'exemple de Hyoung, mais il le devient d'une manière très différente de la créativité linguistique qui encode «l'identité gay » en anglais (Leap 1996 : 12-23) ou encore de l'encodage soigneusement médié qui permet aux « yan daudu du nord du Nigéria d'intégrer une identité d'"homme féminin" à la langue haoussa courante " (Gaudio 2009 : 89-116). De même, au vu de la négociation complexe entre les identités publiques et privées que Hyoung doit mener à bien, mais aussi de la liberté relative qu'il a de se mouvoir entre ces différents lieux, ce que «gay» veut dire en tant qu'inflexion discursive de l'identité sexuelle dans 
l'exemple de cet homme est très différent de ce que ce terme recouvre dans le contexte de l'expérience de Jameson dans le centre-ville du Cap à la fin de l'apartheid (exemple 1).

40 D'autres travaux dans le champ de la linguistique queer sont allés au-delà des tactiques d'intersubjectivité et ont situé les formations individuelles du désir dans le cadre d'une dynamique sociale plus large. Les recherches sur la sexualité, la responsabilité sociale et la «bonne citoyenneté », qui cherchent à savoir la façon dont les sujets qui font état d'une sexualité non conforme sont également en mesure d'adoucir leur aspect potentiellement menaçant vis-à-vis de la société dominante, ont été particulièrement importantes à cet égard. Comme Puar et Rai (2003) l'expliquent, par exemple, lorsque des messages diabolisant l'homosexualité ont émergé aux États-Unis juste après le 11 septembre, ils ont également permis aux « homosexuels » américains de condamner les actes terroristes, en montrant que leur loyauté vis-à-vis de l'État primait sur leur engagement homosexuel ${ }^{9}$. Ces réactions n'ont pas compensé l'augmentation considérable d'agressions physiques ou verbales commises depuis lors contre les homosexuels aux États-Unis et ailleurs.

41 Naturellement, la linguistique queer s'intéresse de plus en plus aux discours homophobes. Tout comme les discours nationalistes, ces discours largement diffusés, assignent quant à eux des significations de mépris, de dégoût ou de haine vis-à-vis de certaines expériences d'homosexualité, qui établissent immédiatement un lien avec d'autres formes de marginalité sociale. Murray définit ainsi l'homophobie comme une "forme de discrimination socialement produite enchâssée dans des relations d'inégalité » (2009: 3), et ne fait aucune référence à la sexualité. Pour Bryant et VidalOrtiz, cet effacement du sexuel pourrait être problématique. Ces deux auteurs estiment que les violences à l'encontre des personnes considérées homosexuelles sont parfois qualifiées d'homophobie, parfois d'agression ou de vol, voire ne sont pas signalées du tout. Contrairement à ses "conceptions et usages considérés comme allant de soi ", l'homophobie n'est pas une formation statique et prédéterminée mais une position discursive dont les usages ont des "conséquences non voulues et parfois loin d'être libératrices » et entraînent souvent leurs " propres formes de violence » (matérielle et discursive) (2008: 391).

\section{La visibilité et les sujets tacites}

L'étude de la formation du sujet tacite menée par Carlos Decena a donné une nouvelle orientation à la linguistique queer. Le sujet tacite est une position discursive observée dans des récits d'hommes dominicains homosexuels vivant à New York. La formation du sujet tacite s'adresse directement à la supposition selon laquelle le sujet, pour se désigner homosexuel, doit être "sorti du placard", visible et explicite quant à son homosexualité. En République dominicaine et dans les lieux où vit la diaspora dominicaine, ce type de déclaration publique est inutile et indésirable, car tout ce qu'il $\mathrm{y}$ a à connaître publiquement de la sexualité d'une personne est déjà connu par les membres de la famille et d'autres proches liés par des relations de dépendance et de respect. Contrairement à ce qui se passe généralement dans le milieu gay aux ÉtatsUnis, les Dominicains homosexuels que Decena a interviewés ont été confrontés à une obligation normative d'" assumer leurs identités sexuelles en négociant le degré avec lequel leurs vies sexuelles et amoureuses deviennent (ou pas) des sujets de discussion 
dans le contexte familial » (Decena, 2008: 340). Ils ont notamment répondu à cette obligation en invoquant les règles de la formation du sujet tacite dans la grammaire espagnole. «Le sujeto tácito est le sujet qui n'est pas formulé mais qui peut être établi à partir de la conjugaison du verbe » ou d'autres moyens linguistiques, explique Decena. "Ce qui est tacite n'est ni secret ni passé sous silence ", bien que cela puisse être déjà compris ou supposé et, "si les gens ont les compétences nécessaires pour reconnaître et décoder [le] comportement ", alors le message tacite n'a pas à être explicité plus avant $(2008: 340)$.

43 Les formations de "sujet tacite » sont attestées chez les hispanophones d'autres pays d'Amérique latine (Wright 2000). Ce sont des principes discursifs de ce type qui guident le processus de "coming out» dans la France urbaine aussi bien que rurale (Provencher 2007 : 85-149), où les hommes et les femmes homosexuels ne disent pas à leurs parents et à leurs amis qu'ils «sont gays » (car ces derniers s'en doutent depuis longtemps et n'ont pas besoin de l'entendre). Ils révèlent plutôt leur implication dans une relation sérieuse avec un partenaire du même sexe - information que les parents et amis n'ont pas forcément saisie (ou n'ont pas voulu saisir) par d'autres moyens. Gabriel, un homme gay de 29 ans, par exemple, raconte cette expérience de coming out vis-à-vis de son père. Gabriel vit à Paris et tient soigneusement sa vie privée à l'écart de sa famille d'origine. Mais un week-end, alors qu'il rend visite à son père (ses parents sont divorcés), au cours d'une discussion dans la soirée, ce dernier soulève le sujet du mariage, de la vie de famille et des enfants. Gabriel poursuit le récit à l'exemple 4 , où l'on voit qu'au lieu d'annoncer clairement «je suis gay », il ajoute une information à une discussion en cours qui force son père à faire la déduction souhaitée : «Non, papa, tu ne comprends pas. Je n'aurai pas d'enfants parce que deux hommes ne peuvent pas avoir d'enfants » $(4: 004-005)$.

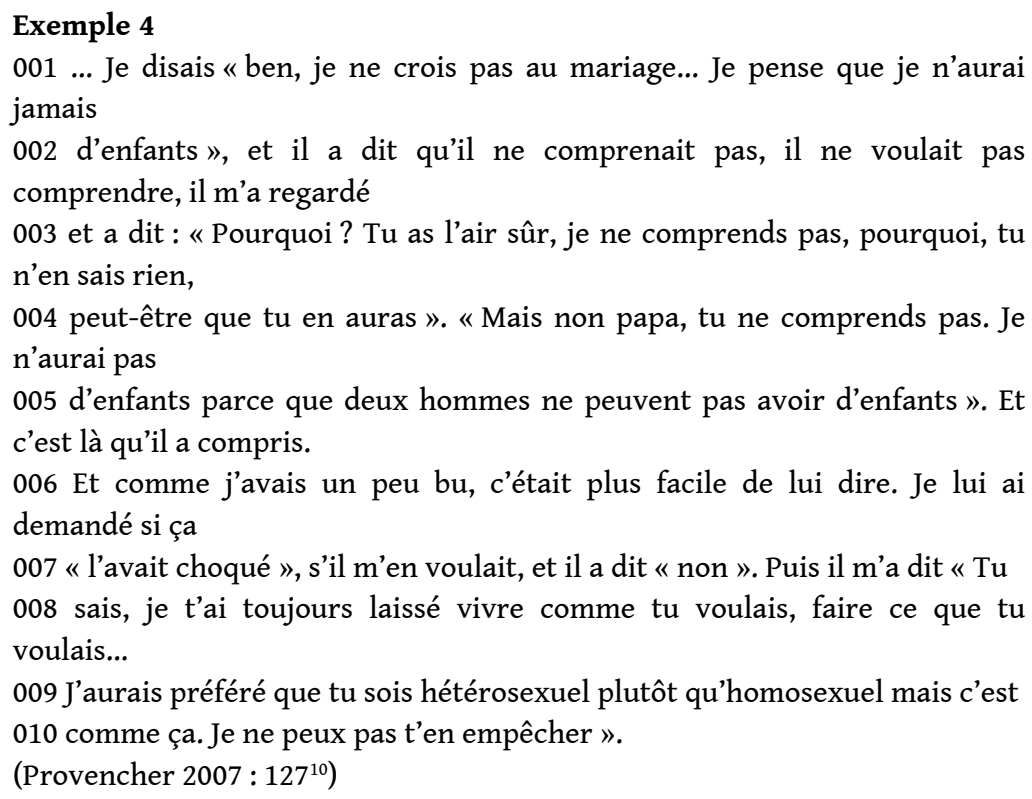

Il est tentant de penser que la réticence de Gabriel à nommer explicitement son statut social est une forme d'allégeance au "placard". Mais ce qui se joue ici, c'est une position discursive tout autre, qui distingue cet exemple français des attentes étasuniennes classiques vis-à-vis du sujet sexuel « homo et fier de l'être » et l'aligne sur la médiation publique de l'homosexualité dans la diaspora dominicaine. 


\section{Les paradoxes de la visibilité : une linguistique queer de la couleur, de la globalisation et de la honte}

Jusqu'à récemment, la linguistique queer était largement basée sur des pratiques discursives visibles et accessibles aux récepteurs et aux chercheurs, ou sur des pratiques discursives qui, pour des raisons données, étaient délibérément cachées. L'intérêt croissant pour les moyens électroniques permettant de communiquer des « messages queer» à la fois localement et dans le monde entier (p. ex. Berry et al. 2003 ; Mowlabocus 2010) a exigé de nouvelles manières d'aborder les tours de parole, la gestion de l'identité, la socialisation langagière et d'autres phénomènes traditionnellement associés à l'interaction orale en face à face. Les nouvelles recherches sur la visibilité ont élargi ces intérêts, et nous ont rappelé que les processus discursifs relevant de la textualité ne sont pas nécessairement explicites. Par conséquent, dans la linguistique queer de même que dans la théorie queer en général, "le décorticage du contenu latent est une tâche aussi importante [pour la théorie queer] que la compréhension de ce qui est ouvertement déclaré » (Giffney 2009 : 7). Dans ces circonstances, des affirmations telles que « si on ne peut pas le dire, alors comment pourrait-on l'être? » - qui a été pendant de nombreuses années la devise informelle de la conférence Lavender Language and Linguistics de l'American University ${ }^{11}$ - revêtent une signification entièrement différente : que veut dire "être " lorsque l'accès aux pratiques discursives associées est médié ou empêché, et non pas aisément accessible?

Cette question ne doit pas être débattue de manière abstraite, mais explorée « in situ ». Ici, les nouveaux travaux de linguistique queer coïncident avec de nouveaux projets de cartographie radicale et de géographie expérimentale (Paglen 2008). Là encore, la linguistique queer rejoint l'ensemble des travaux menés dans le cadre de la «queer of colour critique » (Ferguson 2004 : 1-30). Plus encore que la théorie queer, la queer of colour critique observe la façon dont des attributions de blanchité et de privilège aux racines anciennes dominent les catégories de référence dans les discours populaires et savants, particulièrement lorsque ces discours portent sur l'homosexualité. Le fait que le queer "acquiert sa signification à partir de sa relation d'opposition à la norme" (Halperin 1995: 75) prend un sens supplémentaire, si la "norme» est un terrain discursif privilégié englobant l'hétérosexualité aussi bien que la blanchité, et si la « relation d'opposition» comprend une série de positions dont seulement une partie est formulée explicitement dans la pratique discursive (au sens de l'argument de Decena).

47 Des arguments issus de deux autres domaines de la recherche linguistique queer viennent renforcer la queer of colour critique: les travaux étudiant la diffusion sur la scène mondiale de "l'anglais gay " originaire du continent nord-américain et ceux se penchant sur la façon dont les sujets de la diaspora rencontrent "l'anglais gay" lorsqu'ils arrivent sur le territoire nord-américain (Manalansan 2003; Peña 2004; Boellstorff 2005 ; Leap 2008). Comme sur le plan national, ces discours mondialisés sur l'homosexualité et le privilège soulignent "les imbrications de la race avec la sexualité ». En outre, la rapidité avec laquelle ces discours se diffusent dans le monde évoque les "grandes évasions, les silences et les distorsions des formations nationalistes " (Ferguson, 2009 : 114), même dans un contexte où la question nationale, tout comme la question raciale, devient profondément enchâssée dans 
l'intersubjectivité linguistique et même dans une situation où les désirs ne sont pas satisfaits, en dépit de ces négociations.

Le travail de la linguistique queer consiste également à explorer ces désirs par le biais d'études reliant les pratiques textuelles à des discours plus larges sur la "honte gay » (Mundt 2007; Halperin and Traub 2009) et au "traumatisme queer» (Cvetkovic 2003). Ces recherches se penchent sur des formes de la culture populaire, et vont au-delà de l'intérêt linguistique classique pour le discours verbalisé. En effet, elles explorent de manière plus approfondie les formes d'élaboration de messages qui opèrent avec, à travers et en dépit du langage oral et de l'expression des normes sexuelles qui y sont associées.

\section{BIBLIOGRAPHIE}

ALTHUSSER, Louis. 2011 [1970]. « Idéologie et appareils idéologiques d'État. (Notes pour une recherche) ", in Sur la reproduction. Paris : Presses Universitaires de France, 263-306.

BARRETT, Rusty. 1999. «Indexing polyphonous identity in the speech of African American drag queens ", in Reinventing Identities: The Gendered Self in Discourse, BUCHOLTZ, Mary, LIANG Anita C., SUTTON, Laurel A. (éd.). New York City : Oxford University Press, 313-332.

BERRY, Chris. MARTIN, Fran, and YUE, Audrey (éd.). 2003. Mobile Cultures : New Media in Queer Asia. Durham : Duke University Press.

BOELLSTORFF, Tom. 2005. The Gay Archipelago: Sexuality and Nation in Indonesia. Princeton NJ : Princeton University Press.

BRYANT, Karl, VIDAL-ORTIZ, Salvador. 2008. «Introduction to retheorizing homophobias » Sexualities (Special Issue on Retheorizing Homophobias), 11: 387-396.

BUCHOLTZ, Mary, HALL, Kira. 2004. « Theorizing identity in language and sexuality research » Language in Society, 33: 469-515.

BUTLER, Judith. 2006 [1990]. « Sujets de sexe/genre/désir », in Trouble dans le genre. Le féminisme et la subversion de l'identité. Paris : La Découverte, 59-111. (Traduction Cynthia Kraus)

CAMERON, Deborah, KULICK, Don. 2003a. «Introduction : language and desire in theory and practice », Language and Communication (Special Issue on Language and Desire), 23: 93-105.

CAMERON, Deborah, KULICK, Don. 2003b. Language and Sexuality. Cambridge : Cambridge University Press.

COATES, Jennifer. 2011. "Gender and Discourse Analysis », in The Routledge Handbook of Discourse Analysis, GEE James Paul, HANDFORD Michael (éd.). London : Routledge, 81-113.

CVETKOVIC, Ann. 2003. "Introduction : the everyday life of queer trauma », in An Archive of Feelings: Trauma, Sexuality and Lesbian Public Cultures. Durham : Duke University Press, 15-48.

DECENA, Carlos. 2008. « Tacit subjects » GLQ, A Journal of Lesbian and Gay Studies, 14: 339-359.

ECKERT, Penelope. 2002. « Demystifying sexuality and desire ", in Language and Sexuality: Contesting Meaning in Theory and Practice, CAMPBELL-KIBBLER Kathryn, PODESVA Robert J., 
ROBERTS Sarah J., WONG Andrew (éd.). Stanford : Center for the Study of Language and Information, 99-110.

EDELMAN, Elijah Adiv. 2009. " The power of stealth: (in)visible sites of female-to-male transsexual resistance ", in Out in Public: Reinventing Lesbian/Gay Anthropology in a Globalizing World, LEWIN Ellen, LEAP William L. (éd.). Malden, MA: Wiley-Blackwell, 164-179.

ENG, David L., HALBERSTAM, Jack, and MUÑOZ, José E. 2005. « Introduction : what's queer about queer studies now? » Social Text (Special Issue on What's Queer about Queer Studies Now?), 23: 117.

FAIRCLOUGH, Norman. 2011. "Critical Discourse Analysis », in The Routledge Handbook of Discourse Analysis, GEE James Paul, HANDFORD Michael (éd.). London : Routledge, 9-20.

FERGUSON, Roderick A. 2004. « Introduction : queer of color critique : historical materialism and canonical sociology ", in Aberrations in Black: Toward a Queer of Color Critique. Minneapolis, MN : University of Minnesota Press, 1-31.

FERGUSON, Roderick A. 2009. " The relevance of race for the study of sexuality ", in A Companion to Lesbian, Gay, Bisexual, Transgender, and Queer Studies, HAGGERTY George, MCGARRY Molly (éd.). Malden, MA: Blackwell, 109-123.

GAUDIO, Rudolf Pell. 2009. Allah Made Us : Sexual Outlaws in an Islamic African City. Malden, MA: Wiley-Blackwell.

GIFFNEY, Noreen. 2009. "Introduction : the "q"word ", in Ashgate Research Companion to Queer Theory, GIFFNEY, Noreen, O'Rourke Michael (éd.). Farnham, UK : Ashgate Publishing Ltd, 1-13.

HALL, Kira. 2002. « "Unnatural”gender in Hindi », in Gender Across Languages: The Linguistic Representation of Women and Men, Volume 2, HELLINGER Marlis, BUSSMAN Hadumod (éd.). Amsterdam : John Benjamins, 133-162.

HALPERIN, David M, TRAUB, Valerie (éd.). 2009. Gay Shame. Chicago, IL : University of Chicago Press.

HALPERIN, David. 2000 [1995]. Saint Foucault. Paris : éditions EPEL. (Traduction Didier. Eribon)

KING, Brian W. 2008. " "Being gay guy, that is the advantage": queer Korean language learning and identity construction » Journal of Language, Identity and Education, 7: 230-252.

KULICK, Don. 1998. «Travestí gendered subjectivity », in Travestí. Chicago, IL : University of Chicago Press, 191-238.

KULICK, Don. 1999. « Transgender and language » GLQ: A Journal of Lesbian and Gay Studies, 5: 605622.

KULICK, Don. 2000. « Gay and lesbian language » Annual Review of Anthropology, 29: 243-285.

KULICK, Don. 2003. «Language and desire ", in The Handbook of Language and Gender, HOLMES Janet, MEYERHOFF Miriam (éd.) Malden, MA: Blackwell, 119-142.

LAKOFF, Robin. 1975. Language and Women's Place. New York : Harper and Row.

LEAP, William L. 1996. Word's Out: Gay Men's English. Minneapolis, MN : University of Minnesota Press.

LEAP, William L. 2008. " "True thing that binds us": Globalization, US language pluralism and gay men's English », in The Impact of Globalization on the United States: Culture and Society, BERTHO Michelle (éd.). Westport, CT: Praeger Publishers, 183-209. 
LIVIA, Anna, HALL, Kira. 1997. « “It's a girl!”: bringing performativity back into linguistics », in Queerly Phrased: Language, Gender and Sexuality. New York: Oxford University Press, 3-18.

MANALANSAN, Martin F. 2003. Global Divas: Filipino Gay Men in the Diaspora. Durham : Duke University Press.

MORIEL, Liora. 1998. « Diva in the promised land: a blueprint for newspeak? », World Englishes: Symposium on Creativity in LGBT Discourse, 17: 225-238.

MOWLABOCUS, Sharif. 2010. Gaydar Culture: Gay men, Technology and Embodiment in the Digital Age. Aldershot: Ashgate.

MUNT, Sally R. 2007. Queer Attachments: The Cultural Politics of Shame. Aldershot: Ashgate Publishing.

MURRAY, David A. B. 2009. «Introduction », Homophobias: Lust and Loathing across Time and Space, MURRAY, David A. B (éd.). Durham : Duke University Press, 1-15.

MUÑOZ, José E. 2000. « Introduction : performing disidentifications », in Disidentifications: Queers of Color and the Performance of Politics. Minneapolis, MN : University of Minnesota Press, 1-35.

PAGLEN, Trevor. 2008. « Experimental geography: from cultural production to the production of space », Experimental Geography, THOMPSON Nato. Brooklyn, NY : Melville House Publishing, 2733.

PÊCHEUX, Michel. 1975. « La forme-dujet du discours dans l'appropriation subjective des connaissances scientifiques et de la politique prolétarienne ", in Les vérités de La Palice: linguistique sémantique, philosophie. Paris : Maspero, 196-216.

PEÑA, Susana. 2004. «Pájaration and transculturation: Language and meaning in Miami's Cuban American gay worlds ", in Speaking in Queer Tongues: Globalization and Gay Language, LEAP, William L., BOELLSTORFF, Tom (éd.). Urbana, IL : University of Illinois Press, 231-250.

PROVENCHER, Denis. 2007. Queer French: Globalization, Language and Sexual Citizenship in France. Aldershot: Ashgate.

PUAR, Jasbir K., RAI, Amit S. 2003. « Monster-terrorist-fag: the war on terrorism and the production of docile patriots ", Social Text, 72: 117-148.

SEDGWICK, Eve. 2008 [1990]. «Introduction : Axiomatique », in Épistemologie du placard. Paris : Éditions Amsterdam, 1-66. (Traduction Maxime Cervulle)

VALENTINE, David. 2007. Imagining Transgender: An Ethnography of a Category. Durham : Duke University Press.

VAN DIJK, Teun A. 2011. « Discourse and Knowledge », in The Routledge Handbook of Discourse Analysis, GEE James Paul, HANDFORD Michael (éd.). London : Routledge, 587-603.

WRIGHT, Timothy. 2000. « Gay organizations, NGOs and the globalization of gay identity: the case of Bolivia ", Journal of Latin American Studies, 5: 89-111.

\section{ANNEXES}

\section{Lectures complémentaires}


CAMERON, Deborah, KULICK, Don. 2003. Language and Sexuality. Cambridge : Cambridge University Press.

Un état de l'art utile, bien que parfois partisan, des études sur le langage et la sexualité au cours $\mathrm{du} \mathrm{XX}^{\mathrm{e}}$ siècle, et un argumentaire convaincant en faveur du paradigme du désir pour dépasser les impasses des travaux menés jusque là.

GAUDIO, Rudolf Pell. 2009. Allah Made Us: Sexual Outlaws in an Islamic City. Malden, MA : Wiley-Blackwell.

L'ouvrage explore les pratiques sociales et linguistiques associées à deux formes de masculinité Hausa non-normatives : « yan daudu (des hommes identifiés en premier lieu comme homosexuels, et souvent féminins) et masu harka (des hommes plus masculins, souvent mariés avec des enfants qui "le font" avec des yan daudu). Le livre montre comment le nationalisme Hausa/islamique remodèle le langage, la sexualité et la citoyenneté dans le nord du Nigéria.

KULICK, Don. 2003. « No » Language and Communication (Special issue on Language and Desire), CAMERON, Deborah, KULICK, Don (éd.), 23 (2): 139-151.

Comment l'acte de dire « non » dans le contexte d'une relation hétéro-érotique confirme la position de sujet « femme » - ou confirme une position de sujet « homme » dont la masculinité est alors suspecte.

LEAP, William L. 2008. «Queering gay men's English », in Language and Gender Research Methodologies, HARRINGTON Kate, LITOSSELITI Lia, SAUNTSON Helen, SUNDERLAND Jane (éd.). Basingstoke : Palgrave, 408-429.

Une analyse des récits de coming-out, qui traite l'identité comme un " produit des pratiques linguistiques des locuteurs, plutôt que le fondement sur lequel ces dernières sont basées (2008: 285).

\section{NOTES}

1. NdT: Il s'agit des populations d'ethnies mélangées du Cap. Coloured est une appellation imposée par l'apartheid.

2. Le centre-ville comprend le quartier des affaires ainsi que les quartiers résidentiels et commerciaux adjacents. Ces quartiers étaient réservés aux blancs pendant l'apartheid. Les habitants coloured et noirs ont été transférés dans les townships des Cape Flats, les terres vacantes à l'est du centre-ville.

3. Pendant l'apartheid strict, plusieurs gares ferroviaires étaient des lieux de drague prisés des hommes homosexuels, particulièrement car ces lieux sont des espaces hétérotopiques où les hommes issus de divers milieux raciaux/ethniques pouvaient se rencontrer dans une sécurité relative. Cette tradition s'est perpétuée après l'arrivée de la démocratie.

4. NdT : Théorisée par McRuer (2006), elle vise à critiquer la normalisation du corps valide. " Cripple " est un terme ancien et péjoratif pour désigner les personnes handicapées physiques. Si le terme a été de moins en moins utilisé en anglais au cours $\mathrm{du} \mathrm{XX} \mathrm{X}^{\mathrm{e}}$ siècle dans son sens premier, car jugé trop stigmatisant, il a été peu à peu repris par les activistes afin d'inverser le stigmate, pour mettre en avant une crip culture et organiser des crip prides. (D'après BRASSEUR Pierre. 2016. « Notice : Handicap», in Encyclopédie critique du genre, RENNES Juliette et al (éd.). Paris, La Découverte. 293-305.

5. Voir également le programme de recherche présenté par Kulick (2003 : 130).

6. Une personne trans est stealth lorsqu'elle ne dévoile pas sa transidentité. 
7. Edelman écrit : «Dans la littérature académique, le stealth est essentiellement défini comme la non-divulgation de l'histoire trans présente ou passée d'une personne. Les récits collectés dans le cadre de ce projet montrent que le stealth est une dynamique et une pratique située de négociation idéologique... » (2009: 168-169).

8. Bucholtz et Hall classent leurs tactiques d'intersubjectivité en trois paires: adéquation et distinction, « les processus par lesquels le sujet construit et est construit dans le cadre de la mêmeté et de la différence sociales » $(2004: 494)$; l'authentification et la naturalisation, qui ont à voir avec «la vérité... vs la prétention et l'imposture dans le positionnement identitaire (2004 : 494); et enfin l'autorisation et l'illégitimation, qui distinguent les usages du pouvoir pour légitimer ou refuser une légitimité à des identités sociales (2004:503).

9. De même, le barman du club gay du Cap (exemple 1) a ouvert ce type d'espace pour Jameson et son ami lorsqu'il a demandé : «Vous êtes homos?»

10. Provencher (2007 : 126-127) cite la version française (originale) du texte de Gabriel ainsi que sa traduction. J'ai renuméroté la traduction anglaise dans la présentation de cet exemple.

11. www.american.edu/lavenderlanguages

\section{RÉSUMÉS}

La linguistique queer s'est constituée relativement récemment, mais a très rapidement trouvé sa place parmi les champs académiques qui s'intéressent à l'articulation entre langage, genre et sexualité. De la même manière que les recherches linguistiques sur le genre, la linguistique queer refuse d'aborder les pratiques langagières à travers le prisme de la binarité femme/homme (ou encore à travers un choix réduit d'identités que l'on associe à des pratiques et préférences sexuelles, telles que homo/hétéro). Un des objectifs de la linguistique queer est de révéler les suppositions/idéologies qui amènent les chercheur·euse-s à considérer le genre comme un cadre prédéterminé et figé.

Queer linguistics is a relatively recent academic formation, but one that has quickly become firmly embedded in the current conversations about language, gender, and sexuality. As is also the case in language and gender studies (see Coates, this volume), queer linguistics refuses to frame discussions of linguistic practices in terms of an assumed male/female binary (or on a limited set of identities based solely on erotic practices and preferences). Instead, queer linguistics exposes the assumptions that lead researchers to view gender in terms of a predetermined, static framework.

\section{INDEX}

Thèmes : Recherches

Mots-clés : linguistique queer, analyse du discours, sexualité

Keywords : queer linguistics, discourse analysis, sexuality 


\section{AUTEURS}

\section{WILLIAM L. LEAP}

William L. Leap était professeur d'anthropologie à American University. Ses travaux récents sur le langage et la sexualité explorent les relations inégales entre homosexualité, pratiques

discursives, masculinités et privilèges dans les contexte états-uniens et sud-africain. 